\title{
A numerical method for finding solution of the distributed order time-fractional forced Korteweg-de Vries equation including the Caputo fractional derivative
}

\author{
A. Aminataei ${ }^{1}$ and mohammadhossein derakhshan ${ }^{2}$ \\ ${ }^{1}$ K.N. Toosi University of Technology \\ ${ }^{2}$ K.N.Toosi University of technology
}

August 24, 2020

\begin{abstract}
In this paper, for the first time, the distributed order time-fractional forced Korteweg-de Vries equation is studied. We use a numerical method based on the shifted Legendre operational matrix of distributed order fractional derivative with Tau method to find approximate solution of distributed order forced Korteweg-de Vries equation. This shifted Legendre operational matrix of distributed order fractional derivative with Tau method are used to reduce the solution of the distributed order time-fractional forced Korteweg-de Vries equations to a system of algebraic equations. An error analysis and convergence are obtained. Finally, to display the applicability and validity of the numerical method some examples are implemented.
\end{abstract}

\section{Hosted file}

A new numerical method.pdf available at https://authorea.com/users/353392/articles/477309a-numerical-method-for-finding-solution-of-the-distributed-order-time-fractional-forcedkorteweg-de-vries-equation-including-the-caputo-fractional-derivative 
figures/1a/1a-eps-converted-to.pdf 
figures/1b/1b-eps-converted-to.pdf 
figures/1ea/1ea-eps-converted-to.pdf 
figures/1eb/1eb-eps-converted-to.pdf 
figures $/ 2 a / 2 a-e p s-c o n v e r t e d-t o \cdot p d f$ 
figures/2b/2b-eps-converted-to.pdf 
figures/2ea/2ea-eps-converted-to.pdf 
figures/2eb/2eb-eps-converted-to.pdf 
figures/ap1/ap1-eps-converted-to.pdf 
figures/ap2/ap2-eps-converted-to.pdf 
figures/v11/v11-eps-converted-to.pdf 
figures/v12/v12-eps-converted-to.pdf 